\title{
QUALIDADE E VIDA ÚTIL DE PEQUI MINIMAMENTE PROCESSADO ARMAZENADO SOB ATMOSFERA MODIFICADA
}

\author{
Quality and shelf life of fresh-cut peki stored under modified atmosphere
}

\author{
Éllen Cristina de Souza', Eduardo Valério de Barros Vilas Boas ${ }^{2}$, Brígida Monteiro Vilas Boas ${ }^{3}$, \\ Luiz José Rodrigues ${ }^{4}$, Nélio Ranieli Ferreira de Paula ${ }^{4}$
}

\begin{abstract}
RESUMO
Objetivou-se neste trabalho avaliar a eficiência do uso de atmosfera modificada passiva e ativa na manutenção da qualidade e prolongamento da vida útil de pequi minimamente processado armazenado a $5^{\circ} \mathrm{C}$, por 12 dias, através de avaliações fisiológicas, físicas, físico-químicas e químicas. Os pequis foram lavados em água corrente e sanificados antes e após a retirada da casca em solução de hipoclorito de sódio $200 \mathrm{mg} . \mathrm{L}^{-1} \mathrm{e} 100 \mathrm{mg} . \mathrm{L}^{-1}$, por 15 e 5 minutos, respectivamente. Os pequis foram acondicionados em embalagem rígida de polipropileno (PP), da seguinte forma: coberta com tampa do mesmo polímero; envolta com filme de policloreto de vinila (PVC) $0,014 \mathrm{~mm}$; selada passiva e ativamente $\left(3,2 \% \mathrm{O}_{2}+9,2 \% \mathrm{CO}_{2}\right)$ e $\left(7,7 \% \mathrm{O}_{2}+3,9 \% \mathrm{CO}_{2}\right)$ com filme de polietileno (PE) + PP $0,060 \mathrm{~mm}$. Conclui-se que as embalagens rígidas seladas passiva e ativamente com filme de PE + PP 0,060 mm não são recomendadas para acondicionar pequi minimamente processado, em razão do nível de $\mathrm{O}_{2}$ estar próximo a $0 \%$, no terceiro dia de armazenamento, o que compromete a sua qualidade. As embalagens rígidas de polipropileno com tampa do mesmo polímero e as envoltas com filme de PVC são as mais indicadas para pequi minimamente processado, visto que não propiciam condições de anaerobiose e nem alteraram expressivamente suas características físicas, físico-químicas e químicas, resultando em uma vida útil de 12 dias de armazenamento a $5 \pm 1^{\circ} \mathrm{C}$ e UR $90 \pm 5 \%$
\end{abstract}

Termos para indexação: Caryocar brasiliense Camb., processamento mínimo, armazenamento.

\begin{abstract}
The aim of this work was to evaluate the efficiency of the use of passive and active modified atmosphere on the maintenance of quality and prolongation of the shelf life of fresh-cut peki fruit stored at $5^{\circ} \mathrm{C}$, for 12 days, through physiological, physical, physico-chemistry and chemistry evaluations. Peki fruit were washed with stream water and sanitized with sodium hypochlorite 200 and $100 \mathrm{mg} . \mathrm{L}^{-1}$, for 15 and 5 minutes, before and after peeling, respectively. Peki fruit were packed into rigid propylene package (PP): with rigid cover of same polymer; covered with polyvinyl chloride film (PVC) $0,014 \mathrm{~mm}$; passive and actively $\left(3,2 \% \mathrm{O}_{2}+9,2 \%\right.$ $\left.\mathrm{CO}_{2}\right)$ and $\left(7,7 \% \mathrm{O}_{2}+3,9 \% \mathrm{CO}_{2}\right)$ sealed with polyethylene (PE) + PP film 0,060 mm. One concluded that rigid packages sealed passive and actively with film of PE + PP $0,060 \mathrm{~mm}$ are not recommended to fresh-cut peki fruit condition, due to the fact of the level of $\mathrm{O}_{2}$ to be next to $0 \%$ in the third day of storage, what compromises its quality. The rigid packages of polypropylene with cover of same polymer and those covered with PVC film are the most suitable to fresh-cut peki fruit, since they do not promote anaerobiosis conditions and do not change expressively their physical, physical-chemical and chemical characteristics during 12 days of storage at $5 \pm 1^{\circ} \mathrm{C}$ and $\mathrm{RH} 90 \pm 5 \%$.
\end{abstract}

Index term: Caryocar brasiliense Camb., fresh-cut, storage.

(Recebido em 3 de outubro de 2006 e aprovado em 17 de abril de 2007)

\section{INTRODUÇÃO}

A presença de espécies nativas, com potencial econômico frutífero, na região do Cerrado merece atenção especial, destacando-se o pequizeiro, devido à sua elevada ocorrência nessa região e pelas características sensoriais de seus frutos, marcadas por suas peculiaridades de cor, aroma e sabor, tão apreciadas pela população local. O fruto do pequizeiro ainda constitui-se em fonte de energia, proteínas, fibras, vitaminas, principalmente beta-caroteno e sais minerais (ALMEIDA et al., 1998; RODRIGUES, 2005; VILAS BOAS, 2004). Logo, o processamento mínimo, além de ser uma tecnologia que pode agregar valor ao pequi, pode também contribuir para a expansão do seu consumo, pois esse se restringe quase exclusivamente às regiões em que é produzido e, principalmente, reunir os atributos de

1Doutora em Ciência dos Alimentos - Universidade Federal de Mato Grosso/UFMT - Rodovia MT 100, Km 3,5 - 78698-000 - Pontal do Araguaia, MT ellencsou@hotmail.com

2Doutor, Professor em Ciência dos Alimentos - Departamento de Ciência dos Alimentos/DCA - Universidade Federal de Lavras/UFLA - Cx. P. 3037 37200-000 - Lavras, MG - evbvboas@ufla.br

${ }^{3}$ Doutora em Ciência dos Alimentos - Departamento de Ciência dos Alimentos/DCA - Universidade Federal de Lavras/UFLA - Cx. P. 3037 - $37200-000$ Lavras, MG - bmvboas@hotmail.com

${ }^{4}$ Mestres, Departamento de Ciência dos Alimentos/DCA - Universidade Federal de Lavras/UFLA - Cx. P. 3037 - $37200-000$ - Lavras, MG rodrigues.lui3@uol.com.br; nelioraniel@yahoo.com.br 
conveniência, ou seja, um produto pronto para ser cozido, com qualidade sensorial, valor nutricional e sanidade, tão aclamados pelos consumidores. O mesocarpo interno, polpa carnosa com coloração amarelada, e a amêndoa são as porções consumidas do pequi. O primeiro, cozido, normalmente com outros pratos, como o arroz e o segundo, a amêndoa, assada, depois do aproveitamento do mesocarpo interno (DAMIANI, 2006; RODRIGUES, 2005; VILAS BOAS, 2004).

A vida útil de frutos minimamente processados pode ser prolongada desde que técnicas adequadas de conservação compatíveis com o produto a ser armazenado sejam adotadas. Métodos efetivos de sanificação, o uso do frio e a modificação atmosférica, aliados à qualidade inicial do produto, têm sido usados com sucesso na manutenção dessa qualidade e prolongamento da vida útil de frutos intactos e minimamente processados (VILAS BOAS, 2004).

A modificação da atmosfera de conservação do produto minimamente processado é um dos métodos mais usados para manter a qualidade e, em muitos casos, suplementa a refrigeração. $\mathrm{O}$ ar normal contém $21 \%$ de oxigênio $\left(\mathrm{O}_{2}\right), 0,03 \%$ de dióxido de carbono $\left(\mathrm{CO}_{2}\right), 78 \%$ de nitrogênio $\left(\mathrm{N}_{2}\right)$. Os sistemas de modificação da atmosfera reduzem a concentração de $\mathrm{O}_{2}$ e elevam a de $\mathrm{CO}_{2}$, com o objetivo de diminuir a intensidade da respiração do produto e aumentar a sua vida útil, sem perda da qualidade (CHITARRA, 1998;ZAGORY \& KADER, 1988).

A embalagem de frutos com filmes poliméricos, parcialmente permeáveis ao $\mathrm{O}_{2}, \mathrm{CO}_{2}$ e vapor d'água, constitui-se numa maneira simples de modificação atmosférica. Além de diminuir a taxa respiratória, a embalagem previne o enrugamento e o murchamento do produto, pelo controle da transpiração e evaporação (VILAS BOAS, 2004).

Objetivou-se, neste trabalho, avaliar a eficiência do uso de atmosfera modificada passiva e ativa na manutenção da qualidade e prolongamento da vida útil de pequi minimamente processado armazenado a $5^{\circ} \mathrm{C}$, por 12 dias, através de avaliações fisiológicas, físicas, físicoquímicas e químicas.

\section{MATERIAL E MÉTODOS}

Os pequis foram provenientes da cidade de Itumirim, situada no Sul de Minas Gerais. Os frutos foram colhidos pela manhã, ao acaso, do chão, ou quando ainda unidos à planta-mãe e foram levados para o Laboratório de Pós-Colheita de Frutas e Hortaliças do Departamento de Ciência dos Alimentos da Universidade Federal de Lavras - Lavras, MG.
Os frutos foram selecionados quanto ao grau de maturação (polpa com coloração amarela) e ausência de danos mecânicos ou podridões. Os pequis foram lavados em água corrente com detergente neutro, sanificados em solução de hipoclorito de sódio $200 \mathrm{mg} . \mathrm{L}^{-1}$, por 15 minutos e secos a temperatura ambiente $\left(18^{\circ} \mathrm{C}\right)$. Os caroços foram retirados da casca e posteriormente sanificados em solução de hipoclorito de sódio $100 \mathrm{mg} . \mathrm{L}^{-1}$, por 5 minutos e colocados em peneira plástica, para retirada do excesso de líquido acumulado. Em seguida, foram acondicionados em embalagem rígida de polipropileno (PP) $(15 \times 11,5 \times 4,5 \mathrm{~cm})$ sendo a embalagem:

- fechada com tampa do mesmo polímero;

- envolta manualmente com filme de policloreto de vinila (PVC) $0,014 \mathrm{~mm}$ de espessura;

- selada passivamente com filme flexível de polietileno (PE) + PP alta barreira, 0,060 $\mathrm{mm}$ de espessura, com auxílio da seladora de bandejas TecMaq AP340;

- selada ativamente com filme flexível de PE + PP alta barreira, 0,060 $\mathrm{mm}$ de espessura, com auxílio da seladora de bandejas TecMaq AP340, na qual foi injetada uma mistura de gases inicial contendo $3,2 \% \mathrm{O}_{2}+9,2 \% \mathrm{CO}_{2}$;

- selada ativamente com filme flexível de PE + PP alta barreira, 0,060 $\mathrm{mm}$ de espessura, com auxílio da seladora de bandejas TecMaq AP340, na qual foi injetada uma mistura de gases inicial contendo $7,7 \% \mathrm{O}_{2}+3,9 \% \mathrm{CO}_{2}$.

As embalagens, contendo cerca de $120 \mathrm{~g}$ de frutos, foram armazenadas em câmara fria $\left(5 \pm 1^{\circ} \mathrm{C}\right.$ e UR $\left.90 \pm 5 \%\right)$, por 12 dias e as análises realizadas a cada três dias, sendo as seguintes:

Monitoramento da concentração de $\mathrm{O}_{2}$ e $\mathrm{CO}_{2}(\%)$ no interior da embalagem - foi realizado através de um septo de silicone na superfície do filme plástico, por onde foi retirada uma alíquota da atmosfera interna com auxílio de um analisador de gases PBI Dansensor.

Perda de massa (\%) - foi calculada pela diferença entre a massa inicial dos pirênios contidos dentro das embalagens e a obtida em cada intervalo de armazenamento, utilizando-se balança semi-analítica Mettler, modelo PC2000.

Acidez titulável (\% ácido cítrico) - foi realizada por titulação com solução de $\mathrm{NaOH} 0,01 \mathrm{~N}$, tendo como indicador fenolftaleína, de acordo com o Instituto Adolfo Lutz (1985).

pH - utilizou-se pHmetro TECNAL (Tec 3MP), segundo a técnica da AOAC (1992).

Sólidos solúveis ( ${ }^{\mathbf{B}}$ Brix) - foram determinados em refratômetro digital ATAGO PR-100, com compensação de temperatura automática a $25^{\circ} \mathrm{C}$, segundo a $\mathrm{AOAC}$ (1992). 
Valores $\mathbf{L}^{*}, \mathbf{a}^{*} \mathbf{e} \mathbf{b}^{*}$ - foram feitas em 2 pontos de 5 pirênios, utilizando-se colorímetro marca Minolta, modelo CR 400.

Firmeza (N) - foi realizada em 3 pontos de cada 3 pirênios da repetição. A determinação foi realizada com o auxílio do Texturômetro Stable Micro System modelo TAXT2i, utilizando a sonda tipo agulha P/2N (2 mm de diâmetro), que mediu a força de penetração dela nos pedaços, numa velocidade de $5 \mathrm{~mm} / \mathrm{s}$ e numa distância de penetração de $5 \mathrm{~mm}$, valores esses previamente fixados. Foi usada uma plataforma HDP/90 como base.

$\mathrm{O}$ delineamento utilizado no experimento foi o inteiramente casualizado (DIC), com 3 repetições. Para a variável concentração de $\mathrm{O}_{2}$ e $\mathrm{CO}_{2}$, os tratamentos foram dispostos por um fatorial $5 \times 3$, sendo constituídos pelos fatores atmosfera modificada (tampa, filme de PVC, passiva selada, ativa $3,2 / 9,2$ e ativa $7,7 / 3,9$ ) e tempo de armazenamento ( 0,3 e 6 dias). Já para as demais variáveis, usou-se um esquema fatorial $2 \times 5$, sendo os fatores atmosfera modificada (tampa e filme de PVC) e tempo de armazenamento (0, 3, 6, 9 e 12 dias). A parcela experimental foi constituída por uma embalagem rígida de PP, contendo aproximadamente $120 \mathrm{~g}$ de pequi minimamente processado. As análises estatísticas foram feitas com auxílio do programa Sisvar (FERREIRA, 2000). Após a análise de variância dos dados obtidos, observou-se o nível de significância do teste F.

\section{RESULTADOS E DISCUSSÃO}

Houve interação significativa entre os fatores atmosfera modificada e tempo de armazenamento para as variáveis concentração de oxigênio $\left(\mathrm{O}_{2}\right)$ e dióxido de carbono $\left(\mathrm{CO}_{2}\right)$. De acordo com a Tabela 1 , todas as embalagens com atmosfera modificada utilizadas determinaram diferenças significativas até o sexto dia de armazenamento.

As concentrações de $\mathrm{O}_{2}$ e $\mathrm{CO}_{2}$, logo após a selagem, no interior das embalagens rígidas com tampa, envoltas com filme de policloreto de vinila (PVC) e seladas passivamente encontravam-se semelhantes a do ar atmosférico, enquanto que as concentrações na atmosfera interna das embalagens seladas ativamente $\operatorname{com} 3,2 \% \mathrm{O}_{2}+$ $9,2 \% \mathrm{CO}_{2}$ e $7,7 \% \mathrm{O}_{2}+3,9 \% \mathrm{CO}_{2}$ eram próximas dos valores das respectivas misturas gasosas.

Logo após selagem, a concentração de $\mathrm{O}_{2}$ no interior das embalagens rígidas com tampa, envoltas com filme de PVC e a selada passivamente não diferiu estatisticamente entre si, observando-se valor médio de $20,4 \%$, sendo esse superior ao da embalagem selada ativamente com $3,2 \% \mathrm{O}_{2}$ $+9,2 \% \mathrm{CO}_{2}$ seguido da 7,7\% $\mathrm{O}_{2}+3,9 \% \mathrm{CO}_{2}$. Já no terceiro dia de armazenamento, a concentração de $\mathrm{O}_{2}$ no interior da embalagem com tampa foi superior à das demais, que não diferiram entre si, enquanto, no sexto dia foi superior ao da embalagem envolta com filme de PVC, seguida das embalagens seladas passiva e ativamente.

Tabela 1 - Valores médios de concentração de $\mathrm{O}_{2}$ e $\mathrm{CO}_{2}$ no interior das embalagens rígidas de polipropileno recobertas com diferentes filmes contendo pequi minimamente processado e armazenadas a $5 \pm 1^{\circ} \mathrm{C}$ e UR $90 \pm 5 \%$, por 6 dias.

\begin{tabular}{lccc}
\hline & \multicolumn{3}{c}{ Tempo de armazenamento (dia) } \\
\cline { 2 - 4 } & 0 & 3 & 6 \\
\hline Tampa & $20,4 \mathrm{a}$ & Concentração de $\mathrm{O}_{2}(\%)$ & $19,9 \mathrm{a}$ \\
Filme de PVC & $20,4 \mathrm{a}$ & $20,0 \mathrm{a}$ & $4,0 \mathrm{~b}$ \\
Passiva & $20,4 \mathrm{a}$ & $3,4 \mathrm{~b}$ & $<0,1 \mathrm{c}$ \\
Ativa 3,2/9,2 & $3,2 \mathrm{c}$ & $0,1 \mathrm{~b}$ & $<0,1 \mathrm{c}$ \\
Ativa 7,7/3,9 & $7,7 \mathrm{~b}$ & $<0,1 \mathrm{~b}$ & $<0,1 \mathrm{c}$ \\
\hline & & $0,1 \mathrm{~b}$ & $0,4 \mathrm{~b}$ \\
\hline Tampa & $<0,1 \mathrm{c}$ & $0,4 \mathrm{~b}$ & $6,6 \mathrm{a}$ \\
Filme de PVC & $<0,1 \mathrm{c}$ & $5,8 \mathrm{a}$ & $5,2 \mathrm{a}$ \\
Passiva & $<0,1 \mathrm{c}$ & $6,6 \mathrm{a}$ & $4,7 \mathrm{a}$ \\
Ativa 3,2/9,2 & $9,2 \mathrm{a}$ & $5,4 \mathrm{a}$ & $6,3 \mathrm{a}$ \\
Ativa 7,7/3,9 & $3,9 \mathrm{~b}$ & $6,8 \mathrm{a}$ & $\mathrm{CO}_{2}(\%)$ \\
\hline
\end{tabular}

Médias seguidas de mesma letra na coluna não diferem estatisticamente entre si pelo Teste Tukey $5 \%$. 
A concentração de $\mathrm{CO}_{2}$ no interior da embalagem com tampa, no terceiro e sexto dias, foi inferior que a das demais. No terceiro dia, em diante, de armazenamento, as embalagens rígidas seladas passiva e ativamente determinaram concentração de $\mathrm{O}_{2}$ em torno de $0 \%$, devido provavelmente ao filme plástico ser de alta barreira, o que ocasionou anaerobiose, que é indesejável principalmente sob o ponto de vista da qualidade sensorial e microbiológica. A embalagem rígida com tampa promoveu uma mínima modificação atmosférica.

Damiani (2006) observou que, a despeito do efeito da temperatura, as embalagens rígidas com tampas, contendo pequi minimamente processado, são pouco efetivas quando o objetivo é a modificação interna da atmosfera porque não são fechadas hermeticamente em função do encaixe, permitindo assim trocas gasosas com o meio externo. Em função disso, as concentrações desses gases variaram pouco no interior das embalagens, de 18,8\% a $21 \%$ de $\mathrm{O}_{2}$ e de $0,03 \%$ a $1,53 \%$ de $\mathrm{CO}_{2}$ ao longo do armazenamento.

As embalagens rígidas, com tampa e envoltas com filme de PVC, foram avaliadas até o décimo segundo dia por causa da concentração de $\mathrm{O}_{2}$ no interior das mesmas não ter alcançado níveis próximos a zero. As concentrações de $\mathrm{O}_{2}$ nas embalagens com tampa e envoltas com PVC, diferiram estatisticamente entre si a partir do terceiro dia de armazenamento, sendo que a com tampa determinou a maior porcentagem de $\mathrm{O}_{2}$ (Tabela 2). A porcentagem de $\mathrm{CO}_{2}$ diferiu-se estatisticamente no terceiro, sexto e nono dias em relação às embalagens utilizadas. A embalagem com tampa promoveu baixo acúmulo de $\mathrm{CO}_{2}$, em relação à envolta com PVC.

Pode-se observar que não houve acúmulo de $\mathrm{CO}_{2}$ no interior das embalagens com tampa e envoltas com PVC, ao longo do armazenamento (Tabela 2), visto que, para a maioria das hortaliças minimamente processadas (exceto aquelas que toleram baixos níveis de $\mathrm{O}_{2}$ e altos de $\mathrm{CO}_{2}$ ), uma embalagem adequada deve ser mais permeável ao $\mathrm{CO}_{2}$ que ao $\mathrm{O}_{2}$ (SILVA et al., 2005), cerca de 3 a 5 vezes, dependendo da atmosfera desejada (KADER, 2002).

Em razão do baixo nível de $\mathrm{O}_{2}$, no terceiro dia, dentro das embalagens seladas passiva e ativamente, com filme flexível de PE + PP, apenas as características físicas, físicoquímicas e químicas dos pequis minimamente processados, acondicionados em embalagens rígidas com tampa e envoltas com filme de PVC, foram avaliadas.

Houve interação significativa entre os fatores atmosfera modificada e tempo de armazenamento para a variável perda de massa. A partir do terceiro dia de armazenamento, as embalagens rígidas com tampa determinaram menor perda de massa aos pequis minimamente processados, em relação às envoltas com filme de PVC. No último dia de armazenamento, os pequis acondicionados em embalagens rígidas com tampa apresentaram valores médios de perda de massa de 1,05\% (Tabela 3).

Entretanto, os pequis acondicionados em embalagens envoltas com filme de PVC apresentaram perda de massa na ordem de $1,95 \%$, no final do armazenamento. O mesmo foi notado por Rodrigues (2005), que observou um incremento linear na perda de massa em pequi minimamente processado acondicionado em embalagem envolta com filme de PVC 0,015 $\mathrm{mm}$ ao longo do período de armazenamento a $6^{\circ} \mathrm{C}$, sendo esse aumento mais pronunciado no décimo quinto dia, atingindo $2,16 \%$. De acordo com Vilas Boas (1999), em geral perdas na ordem de $5 \%$ a $10 \%$ são suficientes para causarem um marcante declínio na qualidade em frutos, promovendo perdas na aparência, como o enrugamento do produto.

Tabela 2 - Valores médios de concentração de $\mathrm{O}_{2}$ e $\mathrm{CO}_{2}$, no interior das embalagens rígidas de polipropileno com tampa e envoltas com filme de PVC, contendo pequi minimamente processado e armazenadas a $5 \pm 1^{\circ} \mathrm{C}$ e UR $90 \pm 5 \%$, por 12 dias.

\begin{tabular}{lccccc}
\hline & \multicolumn{5}{c}{ Tempo de armazenamento (dia) } \\
\cline { 2 - 6 } & 0 & 3 & 6 & 9 & 12 \\
\hline Tampa & \multicolumn{5}{c}{ Concentração de $\mathrm{O}_{2}(\%)$} \\
Filme de PVC & $20,4 \mathrm{a}$ & $20,0 \mathrm{a}$ & $19,9 \mathrm{a}$ & $20,2 \mathrm{a}$ & $19,9 \mathrm{a}$ \\
\hline & $20,4 \mathrm{a}$ & $3,4 \mathrm{~b}$ & $4,0 \mathrm{~b}$ & $6,6 \mathrm{~b}$ & $6,7 \mathrm{~b}$ \\
\hline Tampa & \multicolumn{3}{c}{ Concentração de $\mathrm{CO}_{2}(\%)$} & $0,7 \mathrm{a}$ \\
Filme de PVC & $<0,1 \mathrm{a}$ & $0,4 \mathrm{~b}$ & $0,4 \mathrm{~b}$ & $5,6 \mathrm{~b}$ & $2,4 \mathrm{a}$ \\
\hline
\end{tabular}

Médias seguidas de mesma letra na coluna não diferem estatisticamente entre si pelo Teste Tukey $5 \%$. 
Comportamento semelhante foi observado por Leite et al. (2006), que verificaram que a embalagem tipo PET (tereftalato de polietileno), coberta com tampa do mesmo polímero determinou menor perda de massa fresca que a revestida com filme de PVC contendo abacaxi minimamente processado armazenado a $8^{\circ} \mathrm{C}$, por 6 dias. Isso se deve, provavelmente, à característica do filme de $\mathrm{PVC}$ de propiciar maior perda de umidade que PET. A conservação do maior teor de umidade dentro da embalagem favorece a manutenção da turgidez do fruto.

Não houve interação significativa entre os fatores atmosfera modificada e tempo de armazenamento para a variável acidez titulável. Os pequis minimamente processados apresentaram valores médios de acidez titulável de 0,049\% de ácido cítrico. Esse foi menor ao encontrado por Damiani (2006), que observou valor médio de $0,170 \%$ de ácido cítrico, ao estudar o efeito de diferentes temperaturas, na manutenção da qualidade de pequi minimamente processado.

As variáveis sólidos solúveis e $\mathrm{pH}$ foram afetadas significativamente apenas pelo fator tempo de armazenamento. Os teores de sólidos solúveis dos pequis minimamente processados reduziram-se linearmente ao longo do armazenamento (Figura 1A), podendo estar relacionado ao seu consumo no processo respiratório. Rodrigues (2005) também verificou redução de sólidos solúveis, em pequis minimamente processados. Já Damiani (2006) observou um acréscimo no teor de sólidos solúveis. Os valores de $\mathrm{pH}$ dos pequis minimamente processados diminuíram até o sexto dia, em seguida houve um acréscimo até o fim do armazenamento (Figura 1B).

Não houve interação significativa entre os fatores atmosfera modificada e tempo de armazenamento para as variáveis valores $\mathrm{L}^{*} \mathrm{e} \mathrm{a}^{*}$. Os pequis minimamente processados apresentaram valores médios $\mathrm{L}^{*} \mathrm{e} \mathrm{a}^{*}$ de 71,73 e 24,43, respectivamente. Esses valores estão próximos aos encontrados por Damiani (2006), que observou valores médios $\mathrm{L}^{*}$ e a* de 70,69 e 19,05, respectivamente, em pequis minimamente processados mantidos a $5^{\circ} \mathrm{C}$, por 15 dias.

A variável valor $b^{*}$ foi influenciada pela interação significativa entre os fatores atmosfera modificada e tempo de armazenamento. De acordo com a Tabela 4, pode-se observar oscilações no valor $b^{*}$ em pequis minimamente processados acondicionados tanto na embalagem rígida com tampa como nas envoltas com filme de PVC, com tendência de redução ao longo do armazenamento.

Tabela 3 - Valores médios de perda de massa (\%) em pequi minimamente processado acondicionado em embalagens rígidas de polipropileno com tampa, envoltas com filme de PVC e armazenadas a $5 \pm 1^{\circ} \mathrm{C}$ e UR $90 \pm 5 \%$, por 12 dias.

\begin{tabular}{lccccc}
\hline & \multicolumn{5}{c}{ Tempo de armazenamento (dia) } \\
\cline { 2 - 6 } & 0 & 3 & 6 & 9 & 12 \\
\hline Tampa & $0,00 \mathrm{a}$ & $0,25 \mathrm{~b}$ & $0,51 \mathrm{~b}$ & $0,77 \mathrm{~b}$ & $1,05 \mathrm{~b}$ \\
Filme de PVC & $0,00 \mathrm{a}$ & $0,53 \mathrm{a}$ & $1,09 \mathrm{a}$ & $1,49 \mathrm{a}$ & $1,95 \mathrm{a}$ \\
\hline
\end{tabular}

Médias seguidas de mesma letra na coluna não diferem estatisticamente entre si pelo Teste Tukey 5\%.

A

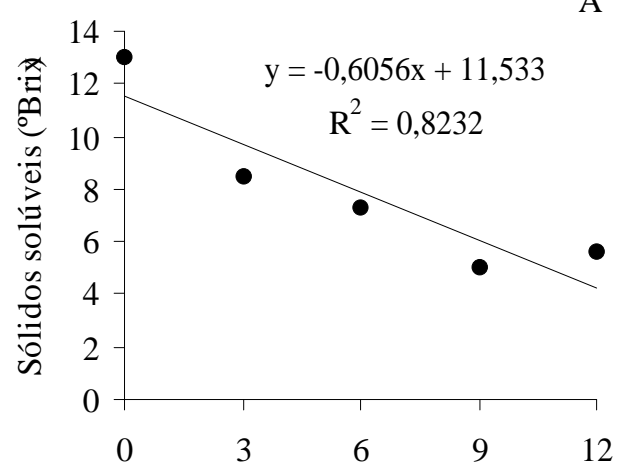

Tempo de armazenamento (dia)
B

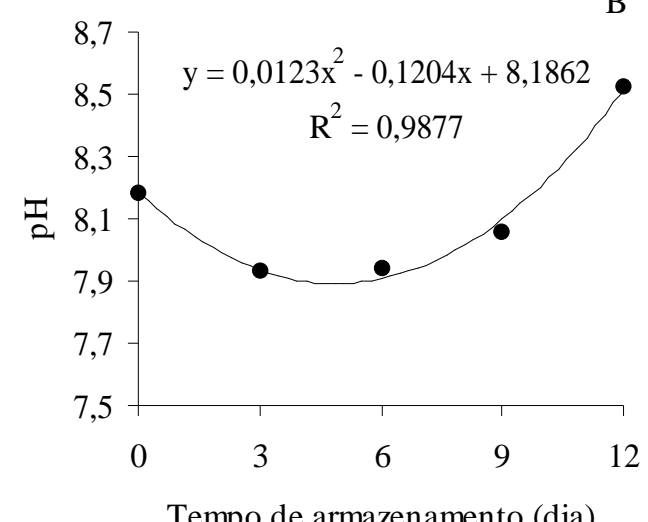

Tempo de armazenamento (dia)

Figura 1 - Valores médios, equações de regressão e coeficientes de determinação de sólidos solúveis (A) e pH (B) em pequi minimamente processado acondicionado em embalagens rígidas de polipropileno com tampa, envoltas com filme de PVC e armazenadas a $5 \pm 1^{\circ} \mathrm{C}$ e UR $90 \pm 5 \%$, por 12 dias. 
Tabela 4 - Valores médios b* em pequi minimamente processado acondicionado em embalagens rígidas de polipropileno com tampa, envoltas com filme de PVC e armazenadas a $5 \pm 1^{\circ} \mathrm{C}$ e UR 90 $\pm 5 \%$, por 12 dias.

\begin{tabular}{lccccr}
\hline & \multicolumn{5}{c}{ Tempo de armazenamento (dia) } \\
\cline { 2 - 6 } & 0 & 3 & 6 & 9 & 12 \\
\hline Tampa & $79,0 \mathrm{a}$ & $79,5 \mathrm{a}$ & $74,3 \mathrm{~b}$ & $77,0 \mathrm{a}$ & $76,5 \mathrm{a}$ \\
Filme de PVC & $79,0 \mathrm{a}$ & $79,3 \mathrm{a}$ & $77,5 \mathrm{a}$ & $76,5 \mathrm{a}$ & $73,7 \mathrm{~b}$ \\
\hline
\end{tabular}

Médias seguidas de mesma letra na coluna não diferem estatisticamente entre si pelo Teste Tukey $5 \%$.

A firmeza não foi afetada significativamente pela interação entre os fatores atmosfera modificada e tempo de armazenamento. Os pequis minimamente processados apresentaram valores médios de firmeza de $5,47 \mathrm{~N}$.

Leite et al. (2006), estudando o efeito de diferentes embalagens nas características físico-químicas de abacaxi 'Smooth Cayenne' minimamente processado, verificaram que a firmeza também não foi afetada significativamente pelos tipos de embalagem utilizadas.

\section{CONCLUSÕES}

Diante dos resultados obtidos pode-se concluir que:

- As embalagens rígidas de polipropileno seladas passiva e ativamente com filme de $\mathrm{PE}+\mathrm{PP} 0,060 \mathrm{~mm}$ não são recomendadas para acondicionar pequi minimamente processado, em razão do nível de $\mathrm{O}_{2}$ estar próximo a $0 \%$ no terceiro dia de armazenamento, o que compromete a sua qualidade.

- As embalagens rígidas de polipropileno, com tampa, do mesmo polímero e as envoltas com filme de PVC são as mais indicadas para armazenar pequi minimamente processado, visto que não propiciam condições de anaerobiose e nem alteraram expressivamente as suas características físicas, físico-químicas e químicas, resultando em uma vida útil de 12 dias de armazenamento a $5 \pm 1^{\circ} \mathrm{C}$ e UR $90 \pm 5 \%$.

\section{REFERÊNCIAS BIBLIOGRÁFICAS}

ALMEIDA, S. P.; PROENÇA, C. E. B.; SANO, S. M.; RIBEIRO, J. F. Cerrado: espécies vegetais úteis. Planaltina: Embrapa-CPAC, 1998. 464 p.

ASSOCIATION OF OFFICIAL AGRICULTURAL CHEMISTS. Official methods of the Association of the Agricultural Chemists. 15. ed. Whashington, 1992. 2 v.

CHITARRA, M. I. F. Processamento mínimo de frutos e hortaliças. Viçosa: UFV, 1998. 88 p.

DAMIANI, C. Qualidade e perfil volátil de pequi (Caryocar brasiliense Camb.) minimamente processado, armazenado sob diferentes temperaturas. 2006. 127 p. Dissertação (Mestrado em Ciência dos Alimentos) - Universidade Federal de Lavras, Lavras, 2006.

FERREIRA, D. F. Análises estatísticas por meio do SISVAR para windows versão 4.0. In: REUNIÃO ANUAL DA REGIÃO BRASILEIRA DA SOCIEDADE INTERNACIONAL DE BIOMETRIA, 45., 2000, São Carlos. Resumos... São Carlos: UFSCar, 2000. p. 235.

INSTITUTO ADOLFO LUTZ. Normas analíticas: métodos químicos e físicos para análise de alimentos. 3. ed. São Paulo, 1985. v. 1, p. 125-181.

KADER, A. A. Modified atmospheres during transport and storage. In: Postharvest technology of horticultural crops. 3. ed. Davis: California, 2002. cap. 14, p. 135-144.

LEITE, D. T. S.; ARTHUR, V.; MATRAIA, C. Efeito de diferentes embalagens nas características físico-químicas de abacaxi 'Smooth Cayenne' minimamente processado. In: ENCONTRO NACIONAL SOBRE PROCESSAMENTO MÍNIMO DE FRUTAS E HORTALIÇAS E HORTALIÇAS, 4., 2006, São Pedro. Palestras, Resumos, Fluxogramas e Oficinas...Piracicaba: USP/ESALQ; CYTED, 2006. p. 136.

RODRIGUES, L. J. O pequi (Caryocar brasiliense Camb.): ciclo vital e agregação de valor pelo processamento mínimo. 2005. 150 p. Dissertação (Mestrado em Ciência dos Alimentos) - Universidade Federal de Lavras, Lavras, 2005.

SILVA, E. de O.; CARNELOSSI, M. A. G.; JACOMINO, A. P.; PUSCHMANN, R.; SOARES, N. de F. F.; ALVES, R. E.; MOSCA, J. L.; FILGUEIRAS, H. A. C.; BASTOS, M. do S. R.; SARRIA, S. D.; YAGUIU, P. Formas de presentación. In: GONZÁlEZ-AGUILAR, G. A.; GARDEA, A. A.; CUAMEA-NAVARRO, F. (Eds.). Nuevas tecnologías de conservación de productos vegetales frescos cortados. Hermosillo: CIAD, 2005. cap. 3, p. 37-58. 
VILAS BOAS, E. V. de B. Aspectos fisiológicos do desenvolvimento de frutos. Lavras: UFLA/FAEPE, 1999. 75 p.

VILAS BOAS, E. V. de B. Frutas minimamente processadas: pequi. In: ENCONTRO NACIONAL SOBRE
PROCESSAMENTO MÍNIMO DE FRUTAS E HORTALIÇAS, 3., 2004, Viçosa. Anais... Viçosa: UFV, 2004. p. 122-127.

ZAGORY, D.; KADER, A. A. Modified atmosphere packaging of fresh produce. Food Technology, Chicago, v. 42, n. 9, p. 70-77, Sept. 1988. 